\title{
Revisiting the Hitherto Elusive Cyclohexanehexone Molecule: Bulk Synthesis, Mass Spectrometry, and Theoretical Studies
}

Dongmei Zhang, ${ }^{+}$Yong Lu, ${ }^{+}$Jie Wang, Chu Gong, Xuesen Hou, Xinxing Zhang, * and Jun Chen*

College of Chemistry, Key Laboratory of Advanced Energy Materials Chemistry (Ministry of Education), Renewable Energy Conversion and Storage Center (RECAST), Frontiers Science Center for New Organic Matter, Nankai University, Tianjin, 300071, China.

\section{Corresponding Authors}

*e-mail: zhangxx@nankai.edu.cn

*e-mail: chenabc@nankai.edu.cn 


\section{Experimental and Theoretical Methods}

Synthetic and characterization methods of $\mathbf{C}_{6} \mathbf{O}_{6}$. The raw material $\mathrm{C}_{6}(\mathrm{OH})_{12} \cdot 2 \mathrm{H}_{2} \mathrm{O}$ was purchased from Shanghai Boka-chem Tech. Inc. The $\mathrm{C}_{6} \mathrm{O}_{6}$ sample was synthesized according to our previous work. ${ }^{1}$ Briefly, $1 \mathrm{~g} \mathrm{C}_{6}(\mathrm{OH})_{12} \cdot 2 \mathrm{H}_{2} \mathrm{O}$ in a $100 \mathrm{~mL}$ flask was heated at $180{ }^{\circ} \mathrm{C}$ in oil bath for $12 \mathrm{~h}$ under continuous vacuum pumping using a mechanic pump to dehydrate $\mathrm{C}_{6}(\mathrm{OH})_{12} \cdot 2 \mathrm{H}_{2} \mathrm{O}$, and the final $\mathrm{C}_{6} \mathrm{O}_{6}$ product was stored in an Ar filled glovebox. The $\mathrm{C}_{6} \mathrm{O}_{6}$ product was further characterized by liquid ${ }^{13} \mathrm{C}$-NMR spectroscopy in a dimethyl sulfoxide-d6 (DMSO-d6) solution using Bruker AVANCE NEO $800 \mathrm{MHz}$ spectrometer. To avoid the contact of $\mathrm{C}_{6} \mathrm{O}_{6}$ with $\mathrm{H}_{2} \mathrm{O}$ and air in the whole process of NMR test, we firstly treated the commercial DMSO-d6 (stored in Ar filled glove box) by activated $3 \AA$ molecular sieve to remove trace $\mathrm{H}_{2} \mathrm{O}$ before use, and added $\mathrm{C}_{6} \mathrm{O}_{6}$ sample and DMSO-d6 in nuclear magnetic tube in Ar-filled glove box, and then the tube was sealed tightly in Ar filled glove box before NMR test.

Electrospray in a glove box. The extended inlet $(3 \mathrm{~cm})$ of an LTQ-XL mass spectrometer (Thermo Fisher Scientific, Waltham, MA, USA) is inserted into a glove box through an UltraTorr fitting attached to the wall of the glove box (Figure S1). The home-built electrospray ionization source is placed $15 \mathrm{~mm}$ away from the MS inlet. A carefully-dehydrated acetonitrile solution of $\mathrm{C}_{6} \mathrm{O}_{6}$ is injected by a mechanical syringe pump through a needle syringe $(500 \mu \mathrm{L}$, Hamilton) to the fused silica capillary $(99.2 \mu \mathrm{m}$ i.d, Polymicro Technologies, US). A coaxial sheath gas $\left(\mathrm{N}_{2}, 120\right.$ psi) flow around the capillary results in nebulization, and helps to direct the spray toward the MS inlet. A high voltage $(-4.5 \mathrm{kV})$ is applied to form the electrospray. The ions of interest are isolated and undergo the collision-induced dissociation at $25 \mathrm{eV}$, and tandem mass spectrometry ( $\left.\mathrm{MS}^{\mathrm{n}}, \mathrm{n}=2-4\right)$ is used to identify the collision fragments.

Theoretical methods. Density functional theory calculations are performed using the Gaussian09 software package. ${ }^{2}$ To better understand $\mathrm{C}_{6} \mathrm{O}_{6}$, we have carried out global minimum (GM) searches by conducting the Coalescence Kick (CK) program. ${ }^{3}$ The geometry optimizations of 10,000 trial structures generated in the $\mathrm{CK}$ program are performed at the B3LYP/6-31G(d) level of theory. ${ }^{4,5}$ Then the eight lowest structures of $\mathrm{C}_{6} \mathrm{O}_{6}$ are reoptimized at a higher level with the aug-cc-pVTZ basis set. ${ }^{6}$ In addition, the structures of $\mathrm{C}_{6} \mathrm{O}_{6} \mathrm{H}^{-}$and $\mathrm{C}_{6} \mathrm{O}_{6}{ }^{2-}$ are also optimized at the B3LYP/aug-cc-pVTZ level of theory. To explore the chemical bonding of $\mathrm{C}_{6} \mathrm{O}_{6}$ and $\mathrm{C}_{6} \mathrm{O}_{6}{ }^{2-}$, the adaptive natural density partitioning (AdNDP) ${ }^{7}$ analyses are performed at the same level of theory to reveal the localized and delocalized chemical bonds. For the ${ }^{13} \mathrm{C}-\mathrm{NMR}$ calculation of $\mathrm{C}_{6} \mathrm{O}_{6}$, the Gauge-Independent Atomic Orbital (GIAO) method is employed. The scaling factors including the slope -1.03 and the intercept 180.75 are applied to obtain scaled chemical shift values. ${ }^{8}$ 


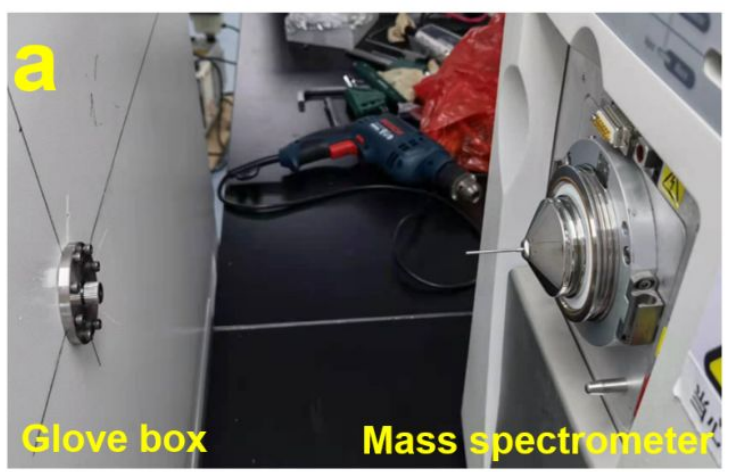

Mass
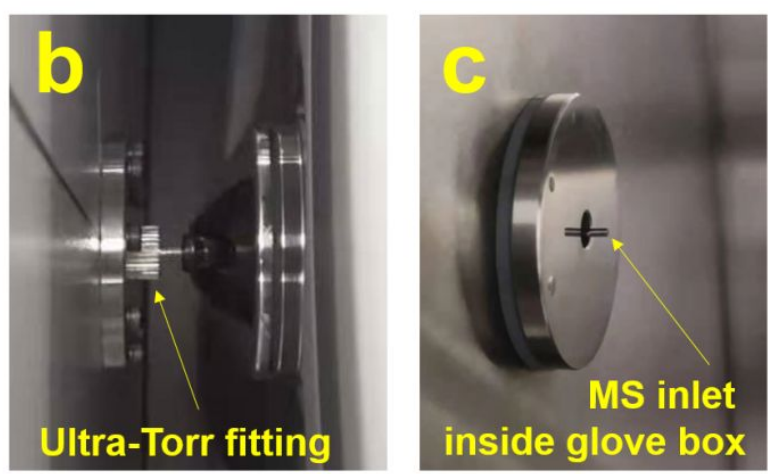

d

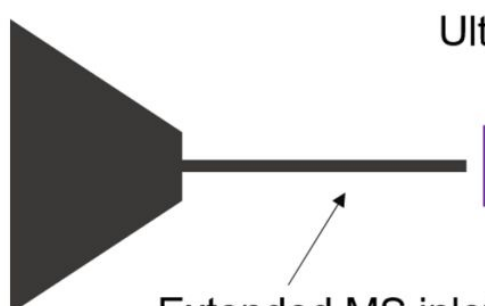

Ultra-Torr fitting

Through hole

Extended MS inlet

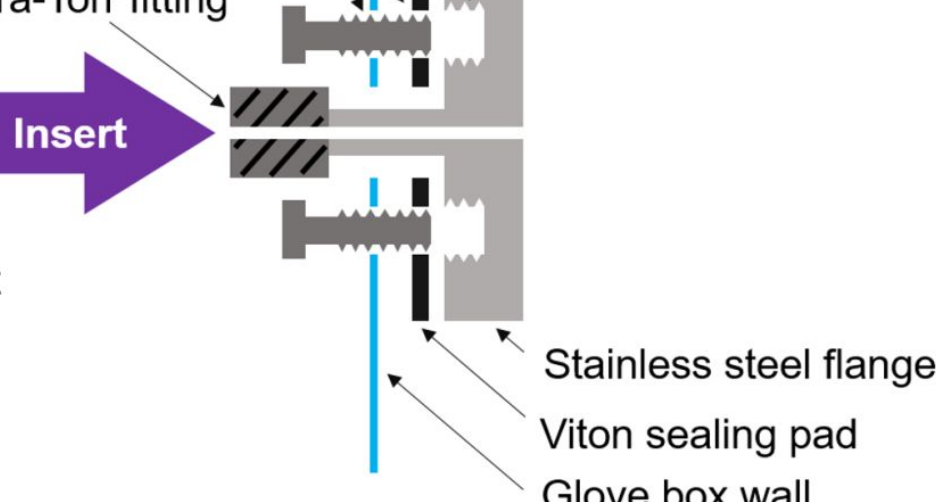

Figure S1. The setup of the electrospray source in a glove box. (a) The glove box and the LTQXL mass spectrometer with a $3 \mathrm{~cm}$ extended inlet. (b) The combination of the mass spectrometer inlet and the glove box through an Ultra-Torr fitting. (c) The inlet of the mass spectrometer in the glove box. (d) A drawing of the overall design. 
a

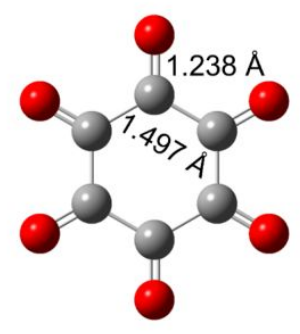

$\mathrm{C}_{6} \mathrm{O}_{6}{ }^{2-} D_{6 h}$ b

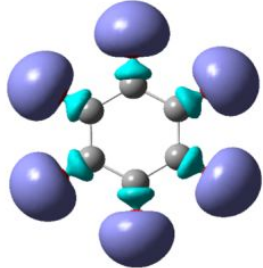

Six 1c-2e lone pairs $\mathrm{ON}=1.98|\mathrm{e}|$

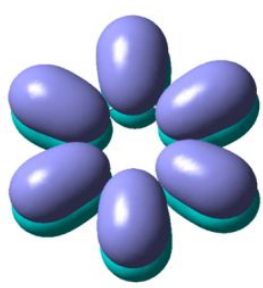

Six $2 \mathrm{c}-2 \mathrm{e} \pi$ bonds $\mathrm{ON}=1.96|\mathrm{e}|$

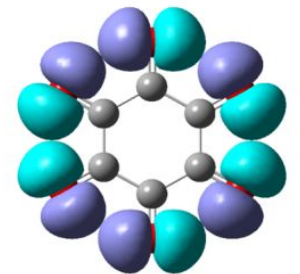

Six $1 \mathrm{c}-2 \mathrm{e}$ lone pairs $\mathrm{ON}=1.89|\mathrm{e}|$

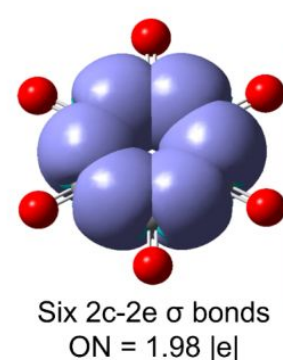

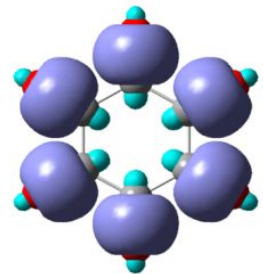

Six $2 \mathrm{c}-2 \mathrm{e} \sigma$ bonds $\mathrm{ON}=1.99|\mathrm{e}|$

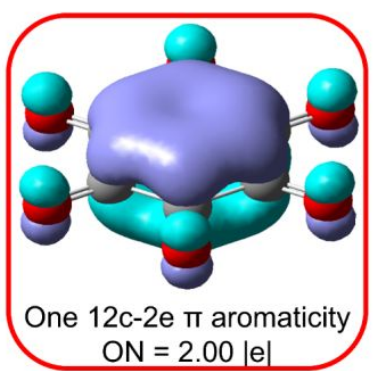

Figure S2. Calculation results of the $\mathrm{C}_{6} \mathrm{O}_{6}{ }^{2-}$ dianion at the B3LYP/aug-cc-pVTZ level of theory. (a) The bond lengths of the planar $D_{6 h} \mathrm{C}_{6} \mathrm{O}_{6}{ }^{2-}$ dianion. (b) The AdNDP analyses of the $\mathrm{C}_{6} \mathrm{O}_{6}{ }^{2-}$ dianion. The first five categories of 2-electron chemical bonds are similar to the neutral $\mathrm{C}_{6} \mathrm{O}_{6}$ molecule, and the bonding circled in the red box presents the delocalized 12c-2e $\pi$ aromaticity, providing an additional stabilizing effect for $\mathrm{C}_{6} \mathrm{O}_{6}{ }^{2-}$ compared to $\mathrm{C}_{6} \mathrm{O}_{6}$. 


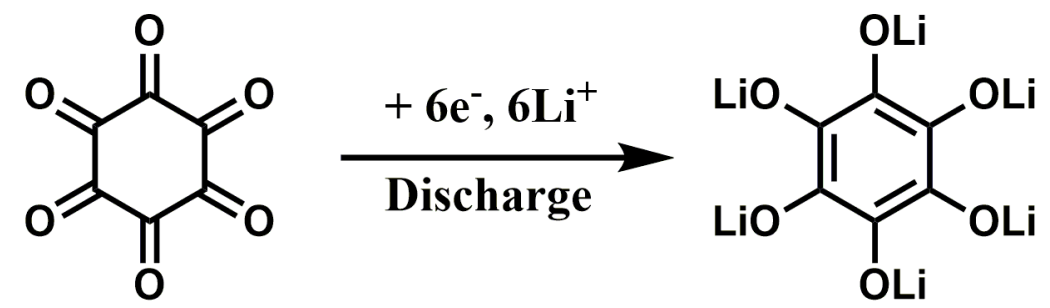

Theoretical capacity: $957 \mathrm{mAh}^{-1}$

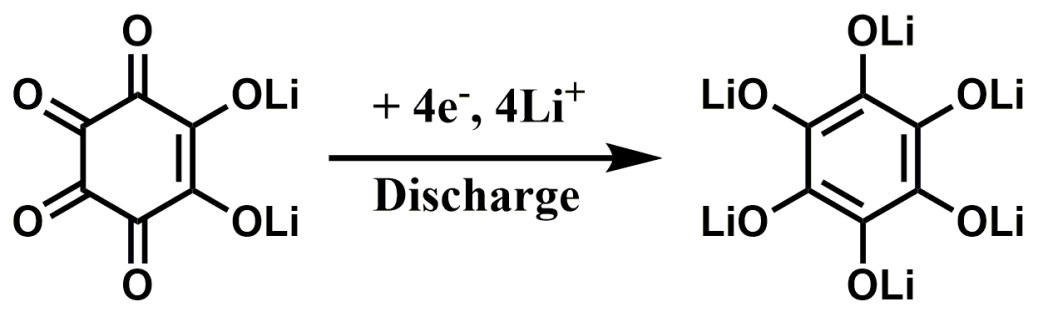

Theoretical capacity: $589 \mathrm{mAh} \mathrm{g}^{-1}$

Figure S3. The discharge reactions and theoretical capacities using $\mathrm{C}_{6} \mathrm{O}_{6}$ and $\mathrm{C}_{6} \mathrm{O}_{6}{ }^{2-}$ as cathode materials in lithium ion batteries. 
Table S1. The calculated 3D coordinates of all the species in the main text at the B3LYP/augcc-pVTZ level of theory.

\begin{tabular}{|c|c|c|c|}
\hline \multicolumn{4}{|c|}{$\mathrm{C}_{6} \mathrm{O}_{6}$ Iso 1} \\
\hline $\mathrm{C}$ & -0.00000800 & -0.66273800 & 0.00010400 \\
\hline $\mathrm{C}$ & -1.40360500 & -1.15579000 & -0.00026900 \\
\hline $\mathrm{C}$ & -1.40354600 & 1.15584300 & 0.00003900 \\
\hline $\mathrm{C}$ & 0.00000700 & 0.66273700 & 0.00002500 \\
\hline $\mathrm{C}$ & 1.40357700 & 1.15581200 & 0.00014100 \\
\hline $\mathrm{C}$ & 1.40357200 & -1.15581900 & 0.00012400 \\
\hline $\mathrm{O}$ & 2.19374500 & -0.00002400 & 0.00014100 \\
\hline $\mathrm{O}$ & 1.85676600 & 2.24906300 & -0.00035300 \\
\hline $\mathrm{O}$ & -1.85675500 & -2.24906900 & 0.00033000 \\
\hline $\mathrm{O}$ & 1.85672500 & -2.24909200 & 0.00058700 \\
\hline $\mathrm{O}$ & -2.19374400 & -0.00000200 & -0.00018000 \\
\hline $\mathrm{O}$ & -1.85673400 & 2.24909000 & -0.00064700 \\
\hline \multicolumn{4}{|c|}{$\mathrm{C}_{6} \mathrm{O}_{6}$ Iso2 } \\
\hline $\mathrm{C}$ & 0.92761900 & 0.00051700 & -0.00017100 \\
\hline $\mathrm{C}$ & 0.24778600 & 1.29494400 & -0.00019800 \\
\hline $\mathrm{C}$ & -1.29827400 & 1.23984800 & 0.00019200 \\
\hline $\mathrm{C}$ & -1.29739600 & -1.24069600 & -0.00074100 \\
\hline $\mathrm{C}$ & 0.24893600 & -1.29469800 & 0.00020600 \\
\hline $\mathrm{C}$ & 2.27544600 & 0.00075600 & -0.00002300 \\
\hline $\mathrm{O}$ & -1.96581800 & -2.21728900 & 0.00016400 \\
\hline $\mathrm{O}$ & 0.80041000 & 2.36514600 & -0.00046000 \\
\hline $\mathrm{O}$ & -1.90883500 & -0.00063000 & -0.00017800 \\
\hline $\mathrm{O}$ & -1.96747000 & 2.21595100 & 0.00070100 \\
\hline $\mathrm{O}$ & 3.41144500 & 0.00086300 & 0.00016000 \\
\hline $\mathrm{O}$ & 0.80218100 & -2.36454400 & 0.00016400 \\
\hline \multicolumn{4}{|c|}{$\mathrm{C}_{6} \mathrm{O}_{6}$ Iso3 } \\
\hline $\mathrm{C}$ & -2.33737100 & 0.24413700 & -0.00020900 \\
\hline $\mathrm{C}$ & -0.13583000 & 1.21798100 & -0.00017800 \\
\hline $\mathrm{C}$ & 1.36709900 & 0.87068800 & 0.00007600 \\
\hline $\mathrm{C}$ & 1.79125500 & -0.61981000 & -0.00020500 \\
\hline $\mathrm{C}$ & -0.56430800 & -1.35490500 & 0.00011000 \\
\hline $\mathrm{C}$ & -1.00524300 & 0.04194100 & -0.00013000 \\
\hline $\mathrm{O}$ & -1.30047900 & -2.29740700 & 0.00036900 \\
\hline $\mathrm{O}$ & -0.50976100 & 2.36472700 & -0.00003400 \\
\hline $\mathrm{O}$ & 2.20278900 & 1.72144000 & 0.00056900 \\
\hline $\mathrm{O}$ & 0.79879500 & -1.58071300 & 0.00023100 \\
\hline $\mathrm{O}$ & -3.45470300 & 0.45044600 & -0.00014300 \\
\hline $\mathrm{O}$ & 2.92665700 & -0.95851700 & -0.00059000 \\
\hline
\end{tabular}




\begin{tabular}{|c|c|c|c|}
\hline \multicolumn{4}{|c|}{$\mathrm{C}_{6} \mathrm{O}_{6} \mathrm{Iso} 4$} \\
\hline $\mathrm{C}$ & 0.81627600 & 1.28931300 & 0.00019900 \\
\hline $\mathrm{C}$ & 1.71686400 & 0.02339600 & 0.00020900 \\
\hline $\mathrm{C}$ & 1.05705000 & -1.38437000 & -0.00009900 \\
\hline $\mathrm{C}$ & -1.13116400 & -0.31742600 & -0.00021500 \\
\hline $\mathrm{C}$ & -0.71331400 & 1.07258700 & -0.00001700 \\
\hline $\mathrm{C}$ & -2.44846300 & -0.57204800 & -0.00030300 \\
\hline $\mathrm{O}$ & 2.90638600 & 0.11007500 & 0.00063200 \\
\hline $\mathrm{O}$ & -0.30815000 & -1.44598400 & -0.00023700 \\
\hline $\mathrm{O}$ & 1.69126300 & -2.38806100 & -0.00011300 \\
\hline $\mathrm{O}$ & -1.47982000 & 2.01134300 & -0.00000600 \\
\hline $\mathrm{O}$ & -3.57896200 & -0.75278800 & -0.00036700 \\
\hline $\mathrm{O}$ & 1.29634500 & 2.38182800 & 0.00026000 \\
\hline \multicolumn{4}{|c|}{$\mathrm{C}_{6} \mathrm{O}_{6}$ Iso5 } \\
\hline $\mathrm{C}$ & -0.91818000 & -1.23297600 & -0.10579500 \\
\hline $\mathrm{C}$ & -1.52682300 & 0.17868300 & 0.10591600 \\
\hline $\mathrm{C}$ & -0.60856400 & 1.41151100 & -0.10618100 \\
\hline $\mathrm{C}$ & 0.91821700 & 1.23287700 & 0.10647600 \\
\hline $\mathrm{C}$ & 1.52676800 & -0.17857700 & -0.10656200 \\
\hline $\mathrm{C}$ & 0.60873800 & -1.41143800 & 0.10614400 \\
\hline $\mathrm{O}$ & 2.66625100 & -0.31196800 & -0.44375400 \\
\hline $\mathrm{O}$ & 1.06329600 & -2.46497900 & 0.44272900 \\
\hline $\mathrm{O}$ & 1.60337500 & 2.15300300 & 0.44381300 \\
\hline $\mathrm{O}$ & -1.60348600 & -2.15353200 & -0.44163300 \\
\hline $\mathrm{O}$ & -1.06285000 & 2.46515000 & -0.44282100 \\
\hline $\mathrm{O}$ & -2.66670400 & 0.31226600 & 0.44166700 \\
\hline \multicolumn{4}{|c|}{$\mathrm{C}_{6} \mathrm{O}_{6}$ Iso6 } \\
\hline $\mathrm{C}$ & -1.83933000 & 0.41147000 & -0.00003700 \\
\hline $\mathrm{C}$ & -0.57604400 & 1.33728800 & -0.00005800 \\
\hline $\mathrm{C}$ & 0.55084800 & 0.39290700 & 0.00012200 \\
\hline $\mathrm{C}$ & 2.01112700 & 0.37667900 & 0.00024400 \\
\hline $\mathrm{C}$ & 0.14717300 & -0.89902600 & 0.00006500 \\
\hline $\mathrm{C}$ & -1.33599900 & -1.07418900 & -0.00001700 \\
\hline $\mathrm{O}$ & -1.98915900 & -2.07300700 & -0.00004200 \\
\hline $\mathrm{O}$ & 2.87666600 & 1.18703000 & -0.00006200 \\
\hline $\mathrm{O}$ & 1.11396100 & -1.79470800 & 0.00001100 \\
\hline $\mathrm{O}$ & -2.97923100 & 0.74924300 & 0.00001300 \\
\hline $\mathrm{O}$ & -0.58885900 & 2.53461300 & 0.00002300 \\
\hline $\mathrm{O}$ & 2.34829200 & -1.01201800 & -0.00018200 \\
\hline \multicolumn{4}{|c|}{$\mathrm{C}_{6} \mathrm{O}_{6}$ Iso 7} \\
\hline $\mathrm{C}$ & 0.22278200 & -0.67132100 & -0.00005500 \\
\hline $\mathrm{C}$ & -0.99599500 & -1.47630700 & -0.00019200 \\
\hline $\mathrm{C}$ & 0.22278400 & 0.67131400 & 0.00001700 \\
\hline
\end{tabular}




\begin{tabular}{|c|c|c|c|}
\hline $\mathrm{C}$ & -0.99598800 & 1.47630900 & 0.00000900 \\
\hline $\mathrm{C}$ & 1.74764100 & 0.80176500 & 0.00011400 \\
\hline $\mathrm{C}$ & 1.74764100 & -0.80177300 & 0.00000600 \\
\hline $\mathrm{O}$ & -2.16962500 & 0.73960800 & -0.00001100 \\
\hline $\mathrm{O}$ & 2.54236900 & -1.67429100 & 0.00001700 \\
\hline $\mathrm{O}$ & -2.16962900 & -0.73959400 & 0.00002400 \\
\hline $\mathrm{O}$ & 2.54237100 & 1.67428200 & -0.00007800 \\
\hline $\mathrm{O}$ & -1.10357700 & -2.66322500 & 0.00010000 \\
\hline $\mathrm{O}$ & -1.10355600 & 2.66323000 & 0.00002500 \\
\hline \multicolumn{4}{|c|}{$\mathrm{C}_{6} \mathrm{O}_{6}$ Iso8 } \\
\hline $\mathrm{C}$ & 0.07206700 & -1.13693700 & 0.00023800 \\
\hline $\mathrm{C}$ & -1.26315800 & -0.38278100 & 0.00010900 \\
\hline $\mathrm{C}$ & -0.96442900 & 1.11656400 & 0.00018800 \\
\hline $\mathrm{C}$ & 0.58042900 & 1.28554100 & 0.00017600 \\
\hline $\mathrm{C}$ & 2.42808700 & -0.36556600 & -0.00030100 \\
\hline $\mathrm{C}$ & 1.11954900 & -0.09296200 & -0.00006000 \\
\hline $\mathrm{O}$ & -2.31831900 & -0.87125600 & 0.74674200 \\
\hline $\mathrm{O}$ & -2.31824400 & -0.87124300 & -0.74660800 \\
\hline $\mathrm{O}$ & 1.16581800 & 2.33253800 & 0.00004000 \\
\hline $\mathrm{O}$ & 0.20345300 & -2.32974700 & 0.00002000 \\
\hline $\mathrm{O}$ & -1.75874400 & 2.00494200 & -0.00018100 \\
\hline $\mathrm{O}$ & 3.54662600 & -0.58312900 & -0.00027500 \\
\hline \multicolumn{4}{|c|}{$\mathrm{C}_{6} \mathrm{O}_{6} \mathrm{H}^{-}$} \\
\hline $\mathrm{C}$ & 1.44716100 & -0.52198700 & -0.12516000 \\
\hline $\mathrm{C}$ & 0.29493800 & -1.50366700 & 0.07630100 \\
\hline $\mathrm{C}$ & -1.08329300 & -0.93829800 & 0.02576300 \\
\hline $\mathrm{C}$ & -1.24721900 & 0.47839000 & -0.05506800 \\
\hline $\mathrm{C}$ & -0.22853700 & 1.48819900 & -0.01379500 \\
\hline $\mathrm{C}$ & 1.19225700 & 0.97213700 & 0.10025500 \\
\hline $\mathrm{O}$ & 0.51579100 & -2.68623100 & 0.24353100 \\
\hline $\mathrm{O}$ & -2.10588200 & -1.65722500 & 0.02971300 \\
\hline $\mathrm{O}$ & -0.45719500 & 2.69556300 & -0.05907600 \\
\hline $\mathrm{O}$ & 2.12162500 & 1.71233500 & 0.33558000 \\
\hline $\mathrm{O}$ & 2.54901400 & -0.91569300 & -0.43655600 \\
\hline $\mathrm{O}$ & -2.52815200 & 0.86861800 & -0.10860800 \\
\hline $\mathrm{H}$ & -3.01343500 & 0.01243600 & -0.08644400 \\
\hline \multicolumn{4}{|c|}{$\mathrm{C}_{6} \mathrm{O}_{6}^{2-}$} \\
\hline $\mathrm{C}$ & -0.00117300 & 1.49641100 & 0.00000000 \\
\hline $\mathrm{C}$ & -0.00035000 & 0.74820700 & -1.29609100 \\
\hline $\mathrm{C}$ & 0.00035000 & -0.74820700 & -1.29609100 \\
\hline $\mathrm{C}$ & 0.00117300 & -1.49641100 & 0.00000000 \\
\hline $\mathrm{C}$ & 0.00035000 & -0.74820700 & 1.29609100 \\
\hline $\mathrm{C}$ & -0.00035000 & 0.74820700 & 1.29609100 \\
\hline
\end{tabular}




\begin{tabular}{lrrr}
$\mathrm{O}$ & -0.00035000 & 1.36750700 & -2.36819900 \\
$\mathrm{O}$ & 0.00035000 & -1.36750700 & -2.36819900 \\
$\mathrm{O}$ & 0.00244000 & -2.73447300 & 0.00000000 \\
$\mathrm{O}$ & 0.00035000 & -1.36750700 & 2.36819900 \\
$\mathrm{O}$ & -0.00035000 & 1.36750700 & 2.36819900 \\
$\mathrm{O}$ & -0.00244000 & 2.73447300 & 0.00000000 \\
\hline
\end{tabular}

\section{References}

(1) Lu, Y.; Hou, X.; Miao, L.; Li, L.; Shi, R.; Liu, L.; Chen, J. Cyclohexanehexone with Ultrahigh Capacity as Cathode Materials for Lithium-Ion Batteries. Angew. Chem., Int. Ed. 2019, 131, 7094-7098.

(2) Frisch, M. J.; Trucks, G. W.; Schlegel, H. B.; Scuseria, G. E.; Robb, M. A.; Cheeseman, J. R.; Scalmani, G.; Barone, V.; Mennucci, B.; Petersson, G. A. Gaussian 09, revision A.1, Gaussian, Inc., Wallingford, CT, 2009.

(3) Sergeeva, A. P.; Averkiev, B. B.; Zhai, H.-J.; Boldyrev, A. I.; Wang, L.-S. All-Boron Analogues of Aromatic Hydrocarbons: $\mathrm{B}_{17^{-}}$and $\mathrm{B}_{18}{ }^{-}$. J. Chem. Phys. 2011, 134, 1-11.

(4) Becke, A. D. Density-Functional Thermochemistry. III. The Role of Exact Exchange. $J$. Chem. Phys. 1993, 98, 5648-5652.

(5) Lee, C.; Yang, W.; Parr, R. G. Development of the Colle-Salvetti Correlation-Energy into a Function of the Electron Density. Phys. Rev. B 1988, 37, 785-789.

(6) Kendall, R. A.; Dunning, T. H.; Harrison, R. J. Electron Affinities of the First-Row and Wave Functions. J. Chem. Phys. 1992, 96, 6796-6806. 
(7) Zubarv, D. Y.; Boldyrev, A. I. Developing Paradigms of Chemical Bonding: Adaptive Natural Density Partitioning. Phys. Chem. Chem. Phys. 2008, 10, 5207-5217.

(8) Konstantinov, I. A.; Broadbelt, L. J. Regression Formulas for Density Functional Theory Calculated1H and13C NMR Chemical Shifts in Toluene-d8. J. Phys. Chem. A 2011, 115, 12364-12372. 